\title{
Management of severe Class II malocclusion with sequential removable functional and orthodontic appliances: A case for MOrthRCSEd* examination
}

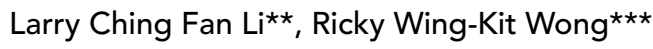

\begin{abstract}
Introduction: Functional appliance is an effective way of treating skeletal Class II malocclusion in children and adolescents. A 12-month stepwise mandibular advancement protocol has been proved to enhance the condylar growth and improve the mandibular prognathism using Herbst appliance. Objective: The following case report documented a 11 year old Chinese girl with $11 \mathrm{~mm}$ overjet treated by a phase I 12-month growth modification therapy using Twin Block appliance with Hyrax palatal expander and high-pull headgear in a stepwise mandibular advancement protocol followed by a phase II preadjusted Edgewise appliance therapy. It is one of the cases submitted for the Membership of Orthodontics Examination of the Royal College of Surgeons of Edinburgh.
\end{abstract}

Keywords: Myofunctional therapy. Functional appliances. Angle Class II malocclusion.

\section{INTRODUCTION}

Based on the incisor relationship, Class II malocclusion is defined as the lower incisor edges lying posterior to the cingulum plateau of the upper incisors resulting in an increase in overjet. ${ }^{1}$ The prevalence of having an overjet greater than $10 \mathrm{~mm}$ was reported to be around $0.2 \%$ of the population. ${ }^{2}$
Large overjet, especially in children and adolescents, is associated not only with an increased risk of traumatic injury to the upper anterior teeth but also psychological distress which results in loss of self-esteem and problems with social interaction. Among different malocclusions, Class II malocclusion was rated as the most unattractive

\footnotetext{
How to cite this article: Li LCF, Wong RWK. Management of severe Class II malocclusion with sequential removable functional and orthodontic appliances: A case for MOrthRCSEd examination. Dental Press J Orthod. 2011 Sept-Oct;16(5):46.e1-11.
}

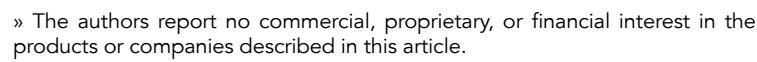
products or companies described in this article. 
by both orthodontists and laypersons. ${ }^{3}$ Albino ${ }^{4}$ assets appearance is the most common reason given for seeking treatments.

Class II malocclusion can usually be corrected by either extracting two upper premolars followed by retraction of the upper anterior teeth (camouflage) or advancing the mandible by growth modification or orthognathic surgery. There are still controversies about how effective is growth modification for the correction of large overjets. Fixed functional appliances such as Herbst has been proven to effectively enhance condylar growth and improve mandibular prognathism in both adolescents ${ }^{5}$ and adults ${ }^{6}$ using a 12 month stepwise mandibular advancement protocol. ${ }^{7}$ The following case report documented a 11 year and 2 month old Chinese girl with $11 \mathrm{~mm}$ overjet treated by a phase I growth modification using Twin Block appliance with the 12 month stepwise protocol followed by a phase II preadjusted Edgewise appliance therapy. It is one of the cases submitted to the Membership of Orthodontics Examination of the Royal College of Surgeons of Edinburgh (MOrthRCSEd).

\section{CASE PRESENTATION}

Clinical examination and diagnostic summary

A 10 year and 10 month old Chinese girl attended our clinic complaining of crooked and sticking out upper anterior teeth. Extraorally, she had no apparent facial asymmetry. The lips were incompetent at rest due to an increase in overjet and also unfavorable muscle tone. The upper lip length was $18 \mathrm{~mm}$, which was considered shorter than the average. There was an acceptable amount of incisal and gingival display on smiling and at rest, with the upper and lower dental midlines coinciding with the midfacial line. The nasolabial angle was acute, reflecting the protrusive upper lip, which together with the retruded mandible and chin, contributed to her convex profile. The temporomandibular joints were normal.
Intraorally, she presented a early permanent dentition with a Class II Division 1, incisor relationship and increased overjet of $11 \mathrm{~mm}$. The overbite was increased at $4 \mathrm{~mm}$ (57\%), complete and traumatic. The molar and canine relationships were full unit Class II on both sides. There was scissorbite between teeth \#14 and \#44. There was mild crowding in the upper arch. The curve of Spee was increased at $3 \mathrm{~mm}$. She also had a reduced anterior Bolton tooth size ratio of $71.3 \%$ (77.7\% for normal Class II Southern Chinese females ${ }^{8}$ ) due to relatively smaller teeth in the lower anterior segment. The oral hygiene needed to be improved (Fig 1).

Radiographically, the increased ANB $\left(6^{\circ}\right)$ and Wits appraisal $(+6 \mathrm{~mm})$ confirmed that the patient had a Class II skeletal pattern. ${ }^{9}$ The normal SNA and reduced SNB and SNPg indicated a normal maxilla, receding mandible and chin. The $\mathrm{SN}$-mandibular plane angle and the lower facial proportion were normal. The upper incisors were proclined while the lower incisors were normally angulated. The lower incisors were far behind the A-Pogonion line and the lower lip was retrusive to the Ricketts E plane by $2.1 \mathrm{~mm}$. The cervical vertebrae maturation (CVM) stage was CVS3, which was around the peak of growth spurt ${ }^{10}$ (Fig 2).

\section{Aims of treatment}

1. Improve oral hygiene.

2. Enhance forward growth of the mandible to improve facial profile and mandible/cranial base relationship.

3. Reduce overjet and overbite and achieve Class I incisor and buccal segment relationships.

4. Relieve crowding and align teeth.

5. Eliminate lip trap and improve lip competency.

Orthodontic treatment comprised a phase I 12-month growth modification therapy using Twin Block appliance with Hyrax palatal expander and high-pull headgear in a stepwise mandibular advancement protocol and a phase II fixed preadjusted Edgewise appliance treatment. 

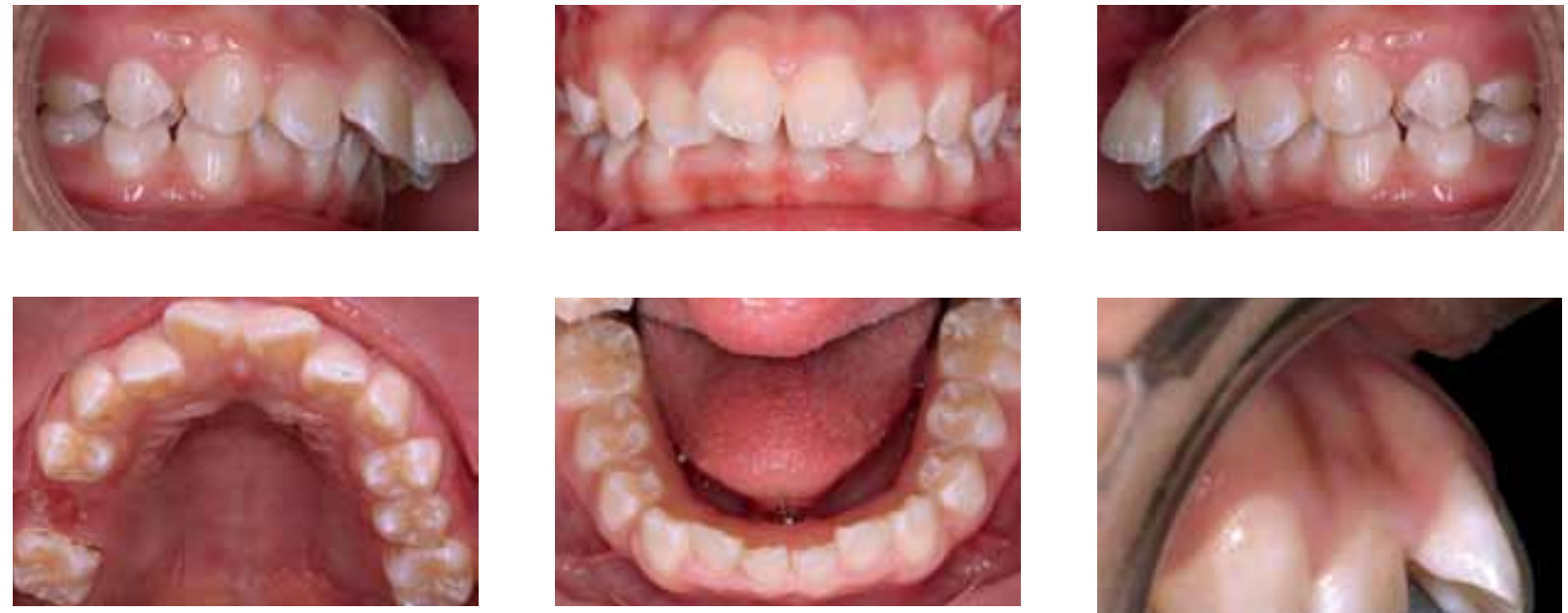

FIGURE 1 - Pretreatment intraoral views.
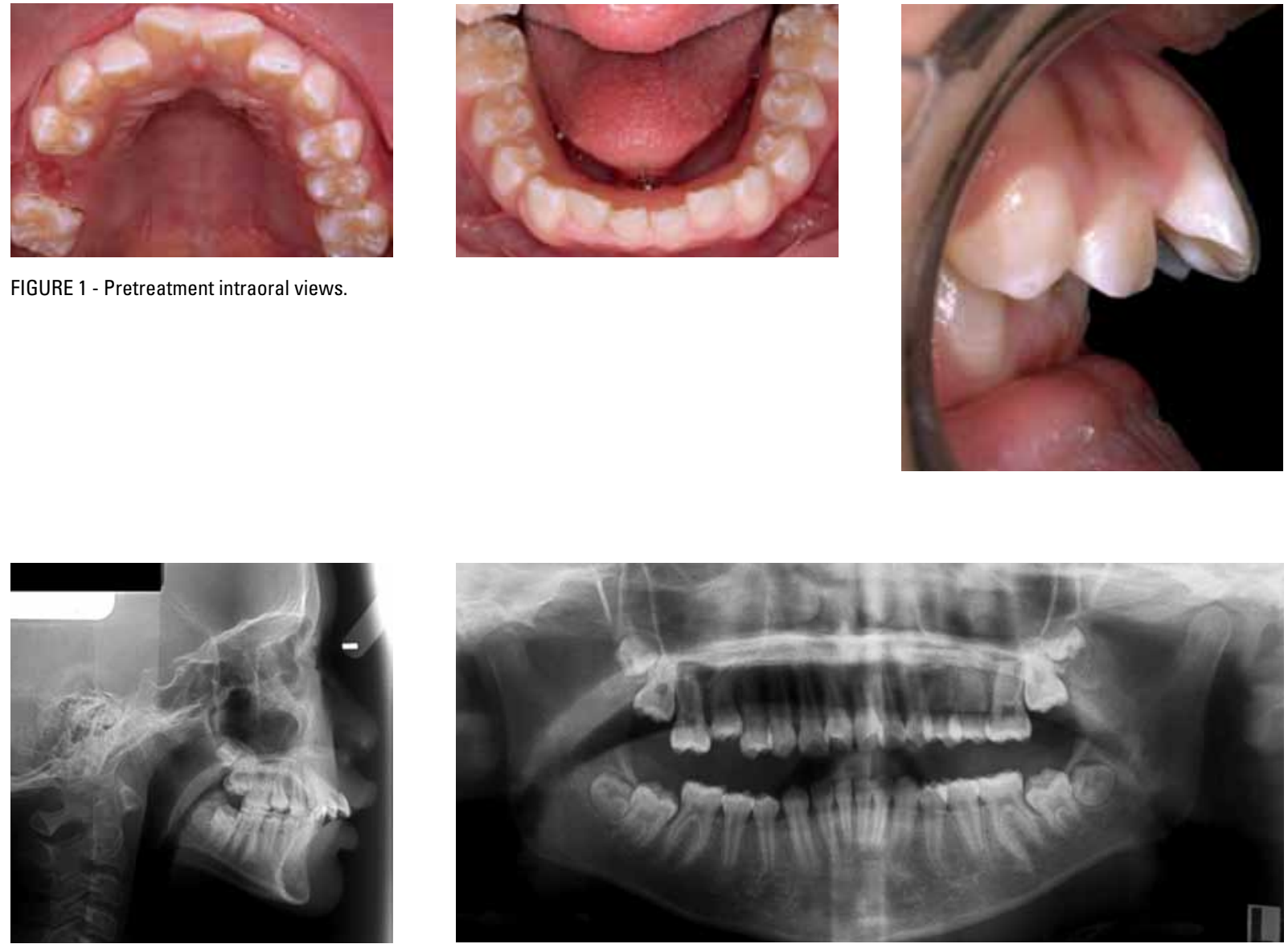

FIGURE 2 - Pretreatment lateral cephalometric and panoramic radiographs.

\section{Treatment progress}

Phase I: Growth modification therapy

The patient was referred to a dental hygienist for oral hygiene instruction and scaling and prophylaxis. After achieving a satisfactory oral hygiene level, orthodontic treatment commenced. An acrylic Twin Block appliance was issued for full-time wear with an initial mandibular advancement of $5 \mathrm{~mm}$ and $7 \mathrm{~mm}$ vertical opening at the premolar area. The appliance was cemented for the first week to ensure initial compliance. A high-pull headgear was issued one month later and was worn with 450 gram of force on each side for 12 to 14 hours per day. After 6 months, the appliance was activated by advancing the mandible another $5 \mathrm{~mm}$ to achieve an edge to edge incisor relationship. At this stage, the Hyrax palatal expander was also activated at a rate of $0.5 \mathrm{~mm}$ per 
week for 12 weeks to achieve a palatal expansion of $6 \mathrm{~mm}$ (Fig 3). Maxillary canine to canine were bonded with 0.014-in nickel-titanium (NiTi) archwire with laceback between teeth \#11 and \#21 to align the teeth.

\section{Phase II: Fixed appliance}

The Twin Block appliance was removed after 12 months into treatment. The overjet was reduced to $1 \mathrm{~mm}$. Crowding was relieved in the upper arch due to distalizing effect of the dentition as well as the palatal expansion. An upper Twin Block retainer providing a positive incisal stop was issued to be worn full-time for 3 months (Fig 4). Both upper and lower arches were bonded using 0.022 × 0.028 -in slot preadjusted Edgewise appliance with Roth's prescription and aligned with 0.014-in nickel-titanium wires. The archwires were subsequently changed to $0.017 \times 0.025$-in NiTi for further alignment
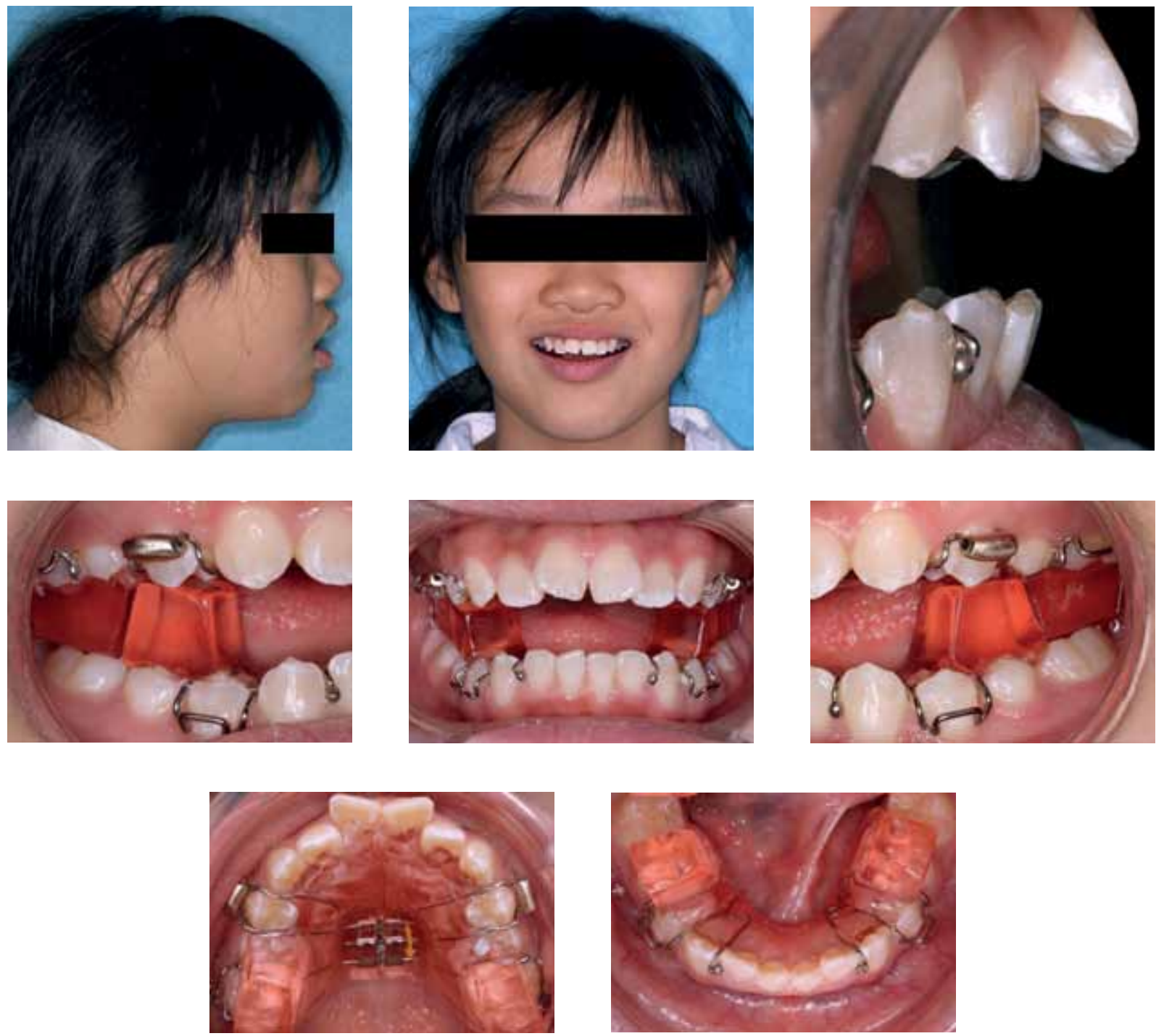

FIGURE 3 - Twin Block appliance bonded. 
and for torque control. After three months, $0.017 \times 0.025$-in stainless steel archwire was placed on the upper arch while $0.019 \times 0.025$-in stainless steel archwire was used in the lower arch for arch coordination. Class II elastics were worn full time to maintain the buccal relationships and the overjet. The treatment was completed in 26 months. Fixed 0.018-in Twisflex fixed lingual retainers were delivered on both arches. Upper and lower Hawley's retainers were also issued as an additional protection measure against unnoticed debonding of the fixed lingual retainers.

\section{Treatment changes}

The total treatment time was 26 months. Overjet and overbite were reduced to $3 \mathrm{~mm}$ and $2.5 \mathrm{~mm}$ respectively. Super Class I buccal relationships were achieved on both sides. Canine guidance was present on the left and right sides during lateral excursions and incisal guidance was present on protrusion. There were no non-working side interferences during function. Protrusive movements were also normal. Good buccal interdigitation was achieved despite buccal overjet was slightly increased due to the tooth size discrepancy. A 97.8\% reduction in PAR (Peer Assessment Rating) score was achieved with the

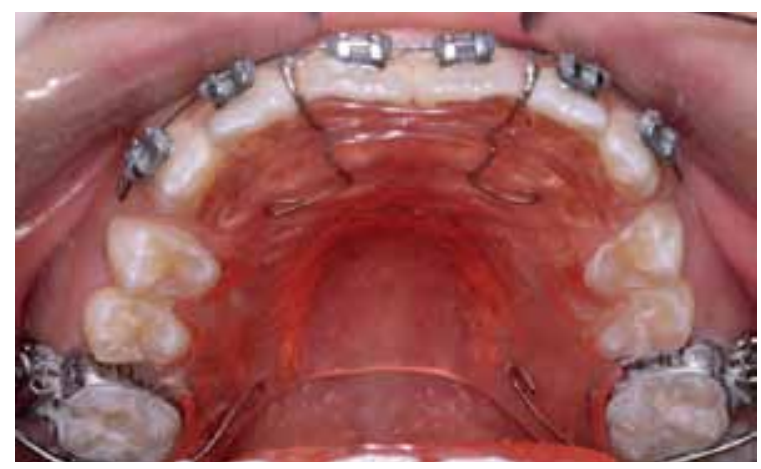

FIGURE 4 - Twin Block retainer. Space developed distal to upper canines due to headgear effect of Twin Block appliance and palatal expansion. initial PAR score of 45 points reduced to 1 point post-treatment. This can be categorized as greatly improved (Figs 5, 6 and 7).

An appreciable amount of sagittal and vertical mandibular growth was observed during the treatment period. The facial profile, measured as facial convexity angle, improved $8^{\circ}$. Mandible/cranial base relationship improved to $3.6^{\circ}$ and Wits appraisal value was at $-2 \mathrm{~mm}$ indicating a Class I skeletal base. ${ }^{9}$ Vertically, the mandibular plane angle was unchanged when compared to pretreatment despite the lower facial height increased by $1.8 \%$. From the superimposition, maxillary growth was restrained during headgear Twin Block treatment, and resumed to a forward and downward pattern during the phase II treatment (Table 1).

Maxillary cephalometric tracings superimposition (Fig 10) indicates that there has been retroclination of upper incisors. Eruption of upper molars was restrained during the headgear/Twin Block treatment, but they were extruded during phase II with fixed appliance treatment.

Mandibular cephalometric tracings superimposition (Fig 10) indicates that there has been very slight lower incisor proclination and maintenance of vertical position, and lower molars extruded and moved mesially during headgear/Twin Block treatment. This explained the development of mild crowding in the lower anterior region after headgear/Twin Block treatment. During the fixed appliance phase, there was continued extrusion of lower molars to level the curve of Spee. The lower incisors were retroclined as a result of rounding off the molar and premolar area. Upward and backward condylar growth was obvious during the overall treatment (Figs 8, 9 and 10).

\section{Sagittal-Occlusion Analysis}

From the Sagittal-Occlusion Analysis (SO-analysis) ${ }^{11}$ immediately after 12 months of headgear/Twin Block treatment, the mandible/cranial base relationship (ss-pg) improved by $7 \mathrm{~mm}$ due to mandibular forward growth (pg/RLp' - pg/RLp) of $6.5 \mathrm{~mm}$, and 

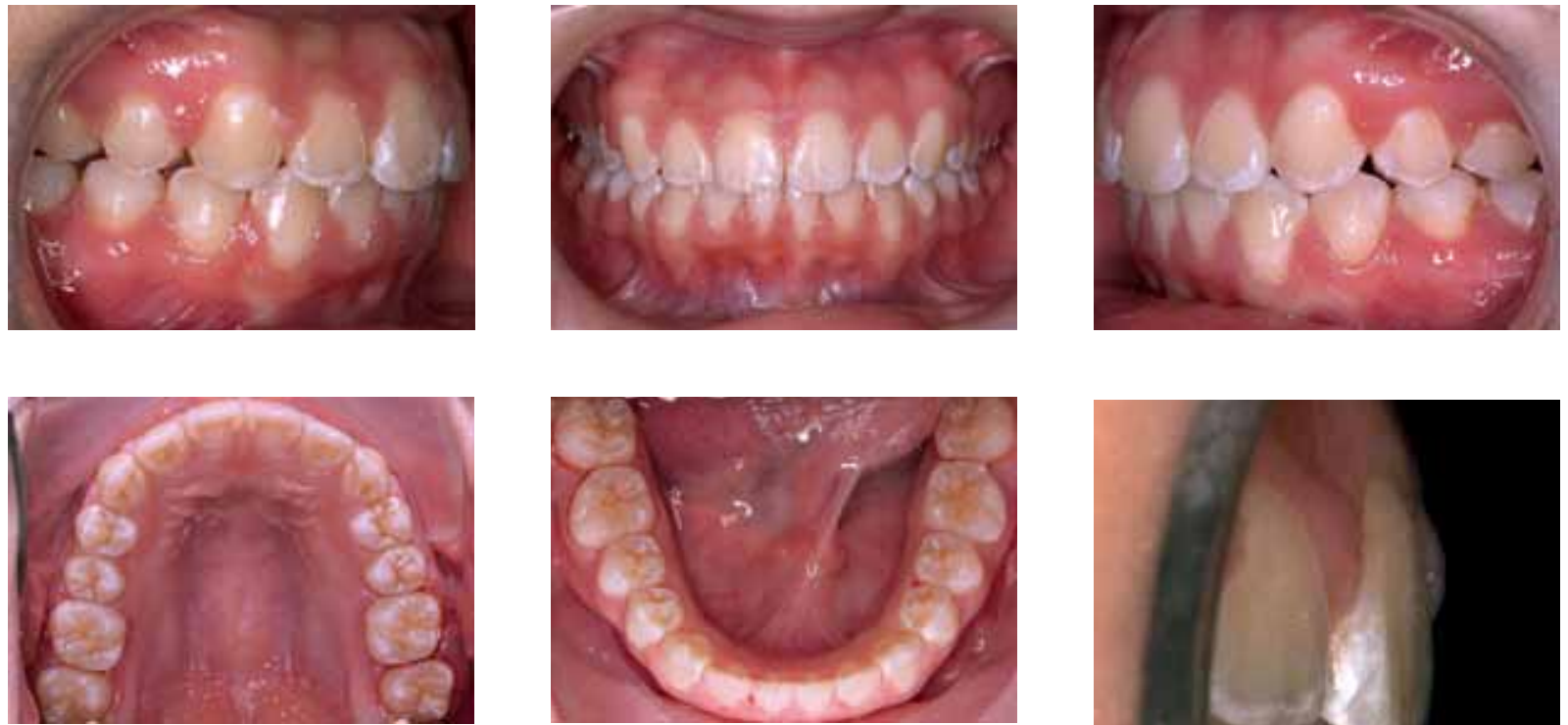

FIGURE 5 - Post-treatment intraoral views.
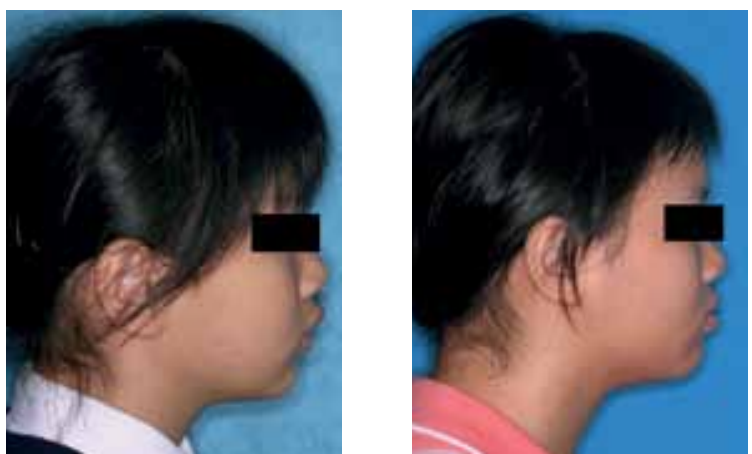

FIGURE 6 - Comparison of pretreatment and post-treatment facial profile.

a maxillary restraint (ss/RLp' - ss/RLp) of $0.5 \mathrm{~mm}$. Maxillary growth restraint was not maintained after phase I, as sagittal maxillary growth caught up by $1 \mathrm{~mm}$ during phase II of treatment. For the mandible, pg-RLp continued to increase although at a much slower rate during phase II, and relapse was minimal according to the analysis.

From the SO-analysis during the headgear/Twin
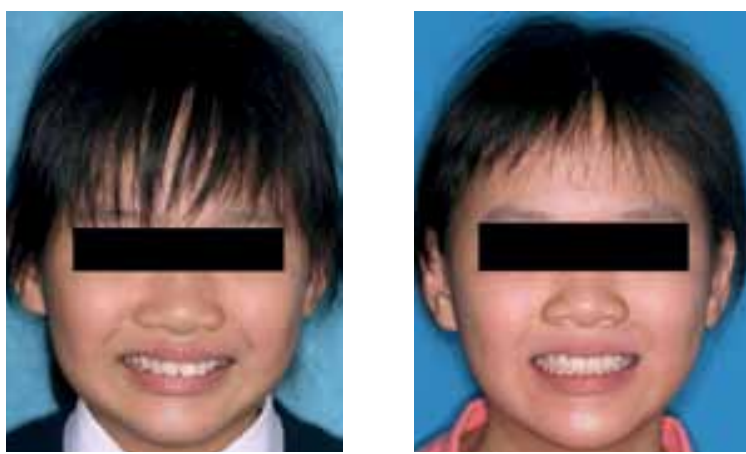

FIGURE 7 - Comparison of pretreatment and post-treatment smile.

Block phase I treatment, the upper incisors were retracted by $3 \mathrm{~mm}$ and the upper molars were distalized by $1.5 \mathrm{~mm}$. The lower incisors were protracted by $0 \mathrm{~mm}$ and the lower molars by $3.5 \mathrm{~mm}$. Overjet improvement of $10 \mathrm{~mm}$ was due to $70 \%$ skeletal and 30\% dental changes, while molar improvement was due to $81 \%$ skeletal and $19 \%$ dental changes (Table 2). 

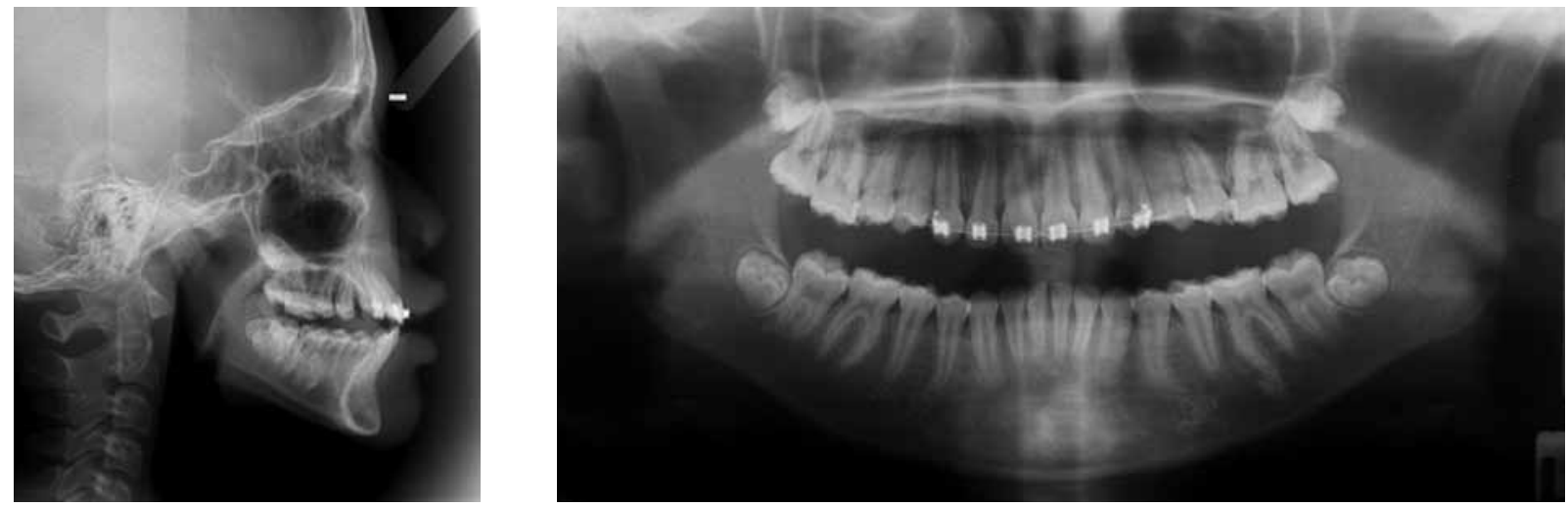

FIGURE 8 - Post-Twin Block lateral cephalometric and panoramic radiographs.
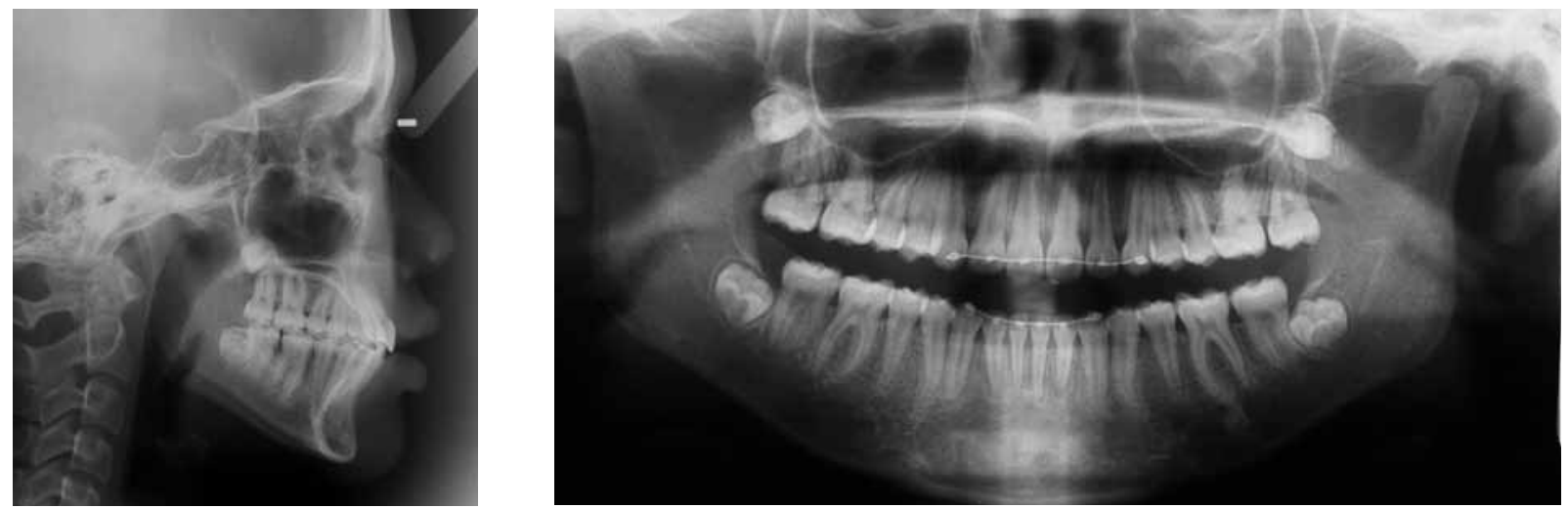

FIGURE 9 - Post-treatment lateral cephalometric and panoramic radiographs.
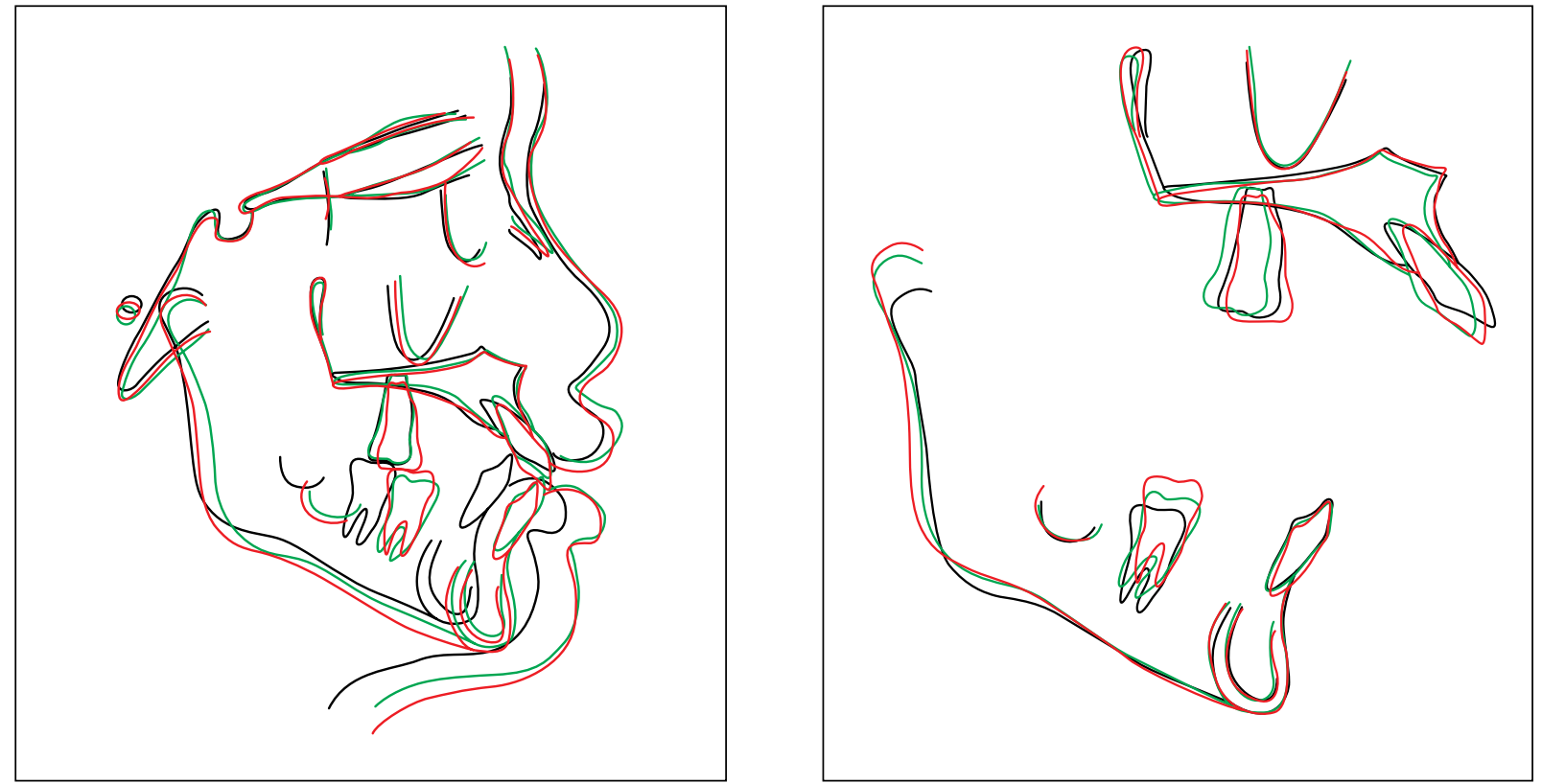

FIGURE 10 - Superimposition of pretreatment, midtreatment and post-treatment cephalometric tracings. 
TABLE 1 - Cephalometric assessment.

\begin{tabular}{|c|c|c|c|}
\hline Variable & Pretreatment & Post-treatment & Change \\
\hline SNA & $80.7^{\circ}$ & $80.9^{\circ}$ & $+0.2^{\circ}$ \\
\hline SNB & $74.1^{\circ}(1 \mathrm{sd})$ & $77.3^{\circ}$ & $+3.2^{\circ}$ \\
\hline SN-Pg & $74.7^{\circ}(1 \mathrm{sd})$ & $78^{\circ}$ & $+3.3^{\circ}$ \\
\hline ANB & $6.6^{\circ}(1 \mathrm{sd})$ & $3.6^{\circ}$ & $-3^{\circ}$ \\
\hline Wits appraisal & $+6 \mathrm{~mm}(2 \mathrm{sd})$ & $-2 \mathrm{~mm}$ & $-8 \mathrm{~mm}$ \\
\hline Upper incisor to maxillary plane angle & $129.7^{\circ}(1 \mathrm{sd})$ & $115.2^{\circ}$ & $-14.5^{\circ}$ \\
\hline Lower incisor to mandibular plane angle & $98.2^{\circ}$ & $94.7^{\circ}$ & $-4.5^{\circ}$ \\
\hline Interincisal angle & $102.4^{\circ}(1 \mathrm{sd})$ & $119.9^{\circ}$ & $+17.5^{\circ}$ \\
\hline SN to mandibular plane angle & $38^{\circ}$ & $38.1^{\circ}$ & $+0.1^{\circ}$ \\
\hline SN to maxillary plane angle & $8,4^{\circ}$ & $7.9^{\circ}$ & $-0,5^{\circ}$ \\
\hline Maxillomandibular plane angle & $29.6^{\circ}$ & $30.2^{\circ}$ & $+0.6^{\circ}$ \\
\hline Upper anterior facial height & $52.6 \mathrm{~mm}$ & $53.2 \mathrm{~mm}$ & $+0.6 \mathrm{~mm}$ \\
\hline Lower anterior facial heigth & $63.7 \mathrm{~mm}$ & $69.5 \mathrm{~mm}(1 \mathrm{sd})$ & $+5.8 \mathrm{~mm}$ \\
\hline Facial height ratio & $54.8 \%$ & $56.6 \%(1 \mathrm{sd})$ & $+1.8 \%$ \\
\hline Lower incisor to Apo line & $+1.6 \mathrm{~mm}(1 \mathrm{sd})$ & $+4.9 \mathrm{~mm}$ & $+3.3 \mathrm{~mm}$ \\
\hline Lower lip to Ricketts E plane & $1.9 \mathrm{~mm}$ & $2.6 \mathrm{~mm}$ & $+0.7 \mathrm{~mm}$ \\
\hline Me-Go: S-N ratio & $68: 68$ & $73: 69$ & \\
\hline
\end{tabular}

Note that the norm values were based on a sample of 12 year old children.

Note: All values above have been adjusted to the SN-Frankfort Horizontal plane.

Sources of normal values for chinese:

Cooke MS and Wei SHY(1988) Eur J Orthod. 10(3):264-72

Cooke MS (1986) Ph. D Thesis. the University of Hong Kong. Cephalometric analyses based on natural head posture of children in Hong Kong

$(1 \mathrm{sd})=$ One standard deviation

$(2 \mathrm{sd})=$ Two standard deviation

\section{DISCUSSION}

\section{Treatment rationale}

In many respects the patient was an ideal candidate for functional appliance treatment. She presented with a mild to moderate Class II skeletal discrepancy, average vertical dimensions, mild crowding and proclined upper incisors, with the lower incisors presenting average inclination. The functional appliance was used to correct the skeletal discrepancy, and correct incisor and buccal segment relationships to Class I. As a result of the potential skeletal and dentoalveolar changes produced by the functional appliance, a more favorable soft tissue environment was created with elimination of the lip trap and the lower lip acting labially on the upper incisors.
Orthodontic camouflage by extraction of upper premolars could have been another treatment option but was not considered for a number of reasons. The patient and her mother were keen to avoid extractions due to concerns about removing healthy teeth. Extraction of upper premolar teeth might be able to retract the upper protrusive lip and improve facial convexity to certain extent, but would not improve mandibular retrognathism. Extraction approach also required careful anchorage management, which in her case, might involve headgear or bone anchorage, and thus carry other potential problems such as incompliance and patient discomfort.

Removable functional appliance was used in this patient because her premolars were not fully 
erupted yet when the treatment started. Otherwise, Herbst appliance would be another option because we could minimize any potential compliance problem ${ }^{12}$ and maximize treatment efficiency. The success of the treatment was largely dependent on the patient's compliance which was very well accomplished in this case. Mandibular advancement every six months in a stepwise manner has been proved more effective in stimulating condylar growth ${ }^{13}$ and improving mandibular prognathism. ${ }^{14}$ The use of high-pull headgear during the functional appliance stage helped restraining the maxillary growth and also prevented clockwise rotation of the maxilla which might cause backward and downward rotation of the mandible thus jeopardizing the treatment effects. ${ }^{15}$

FIGURE 11 - Sagittal Occlusion Analysis.

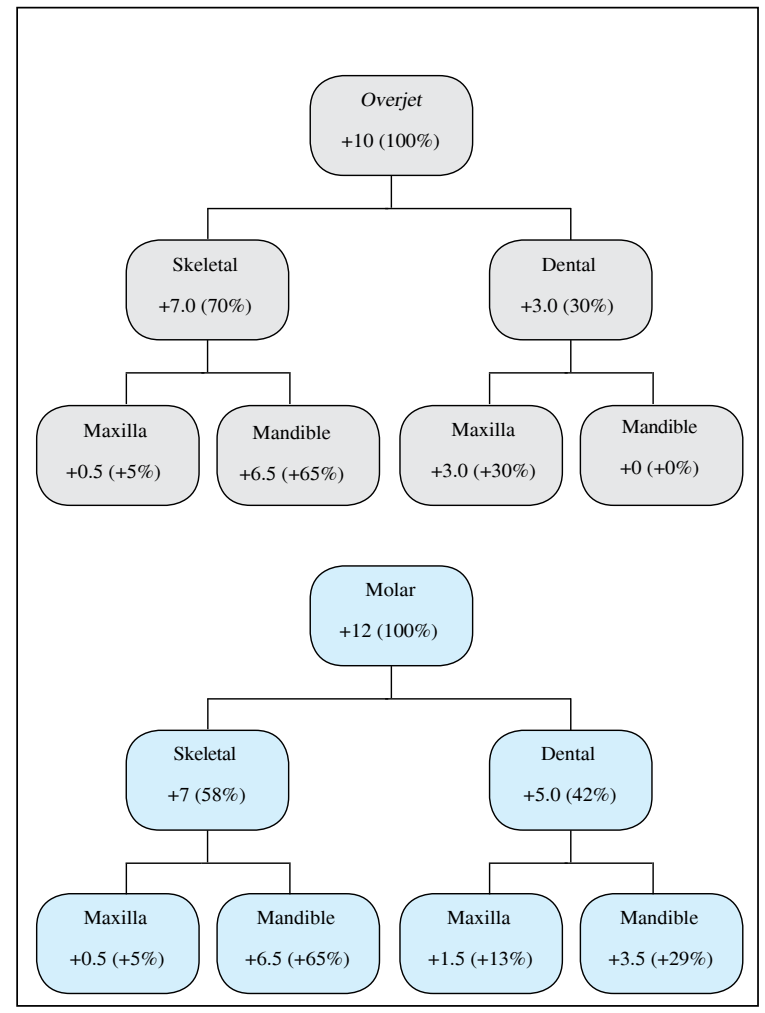

Source of analysis: Pancherz, ${ }^{11} 1982$.
Lower incisor proclination is one of the major side effects of functional appliances. In this case, the proclination of the lower incisors was minimized to only $0.6^{\circ}$. This common side effect in using functional appliance was avoided by trimming away the acrylic lingual to the lower anterior teeth. The fact that patient had smaller lower incisors and also the pressure from the lower lip might also have helped maintaining the angulations of the lower incisors.

\section{Two phase vs. one phase treatment}

The total treatment time was 26 months including 12 months of growth modification. The main aim of the two phase orthodontic treatment was to enhance the patient's potential for favourable mandibular growth and improve her skeletal and soft tissue profile by growth modification. It was also planned to avoid over-retraction of her upper incisors with respect to the incisor angulations, future nasal growth and her existing smile line. The decision to start treatment with a first phase of functional appliance was proved to be appropriate.

The patient had her menarche in the sixth month during the Twin Block phase treatment, indicating that she was around the peak of her pubertal growth spurt, ${ }^{16}$ which was corresponding to her CVM stage. ${ }^{17}$ The treatment effect by the functional appliance could be maximized during this period. ${ }^{18}$

The lip competency improved although was not fully corrected due to unfavourable muscle tone. There was increased buccal overjet as a result of tooth size discrepancy between upper and lower arches. It was decided not to change the size and shape of the teeth by stripping or building-up with resin because of esthetic concerns. There was mild generalized gingival swelling at the interdental papilla area, despite fair oral hygiene had been maintained by the patient during treatment. The gingival swelling resolved greatly one week after debonding. 


\section{Long term prognosis}

The mandibular intercanine width has been maintained at its original while the upper was expanded during the functional appliance phase. The prognosis for stability is good provided the patient's growth pattern is favourable and the mandible will not rotate downward and backward. Good buccal interdigitation and incisal contact also helped to stabilize the occlusal stability, as well as the fixed and removable retainers.

\section{CONCLUSION}

A number of researches have shown that a 12-month stepwise mandibular advancement protocol using headgear/Herbst appliance could enhance the condylar growth and improve the mandibular prognathism. This case report documented a successful treatment of a skeletal Class II using removable headgear/Twin Block appliance with the 12-month stepwise advancement protocol. The long-term clinical effects of such treatment protocol demand a case-controlled study with more samples and longer follow up period. 


\section{REFERENCES}

1. Mitchell L, Carter NE, Doubleday B. An introduction to Orthodontics. $2^{\text {nd }}$ ed. Oxford: Oxford University Press; 2001.

2. Proffit WR, Fields HW Jr, Sarver DM. Contemporary Orthodontics. $4^{\text {th }}$ ed. Missouri: Elsevier; 2007.

3. Cochrane SM, Cunningham SJ, Hunt NP. Perceptions of facial appearance by orthodontists and the general public. J Clin Orthod. 1997 Mar;31(3):164-8.

4. Albino JE. A psychologist's guide to oral diseases and disorders and their treatments. Prof Psychol Res Pract. 2002;33(2):176-82.

5. Hägg U, Du X, Rabie AB. Initial and late treatment effects of headgear-Herbst appliance with mandibular step-bystep advancement. Am J Orthod Dentofacial Orthop. 2002;122(5):477-85.

6. Purkayastha SK, Rabie AB, Wong R. Treatment of skeletal Class II malocclusion in adults: stepwise vs single-step advancement with the Herbst appliance. World J Orthod. 2008 Fall;9(3):233-43.

7. Rabie AB, She TT, Hägg U. Functional appliance therapy accelerates and enhances condylar growth. Am J Orthod Dentofacial Orthop. 2003 Jan;123(1):40-8.

8. Ta TA, Ling JY, Hägg U. Tooth-size discrepancies among different occlusion groups of southern Chinese children. Am J Orthod Dentofacial Orthop. 2001;120(5):556-8.

9. Cooke MS, Wei SHY. Cephalometric standards for the southern Chinese. Eur J Orthod. 1988;10:264-72.

10. Baccetti T, Franchi L, James A, McNamara JA Jr. The cervical vertebral maturation (CVM) method for assessment of optimal treatment timing in dentofacial orthopaedic. Semin Orthod. 2004;11:119-29.
11. Pancherz $\mathrm{H}$. The mechanism of Class II correction in Herbst appliance treatment. A cephalometric investigation. Am J Orthod. 1982;82(2):104-13.

12. O'Brien K, Wright J, Conboy F, Sanjie Y, Mandall N, Chadwick S, et al. Effectiveness of treatment for Class II malocclusion with the Herbst or Twin-Block appliances: a randomized, controlled trial. Am J Orthod Dentofacial Orthop. 2003;124(2):128-37.

13. Bakr A, Rabie AB, Al-Kalaly A. Does the degree of advancement during functional appliance therapy matter? Eur J Orthod. 2008;30(3):274-82.

14. Hägg U, Rabie AB, Bendeus M, Wong RW, Wey MC, Du $X$, et al. Condylar growth and mandibular positioning with stepwise vs maximum advancement. Am J Orthod Dentofacial Orthop. 2008 Oct;134(4):525-36.

15. Du X, Hägg U, Rabie AB. Effects of headgear Herbst and mandibular step-by-step advancement versus conventional Herbst appliance and maximal jumping of the mandible. Eur J Orthod. 2002;24(2):167-74.

16. Hägg U, Taranger J. Maturation indicators and the pubertal growth spurt. Am J Orthod. 1982;82(4):299-309

17. Al Khal HA, Wong RW, Rabie AB. Elimination of hand-wrist radiographs for maturity assessment in children needing orthodontic therapy. Skeletal Radiol. 2008;37(3):195-200. Epub 2007 Oct 3.

18. Hägg $U$, Pancherz H. Dentofacial orthopaedics in relation to chronological age, growth period and skeletal development. An analysis of 72 male patients with Class II division 1 malocclusion treated with the Herbst appliance. Eur J Orthod. 1988;10(1):169-76.

\section{Contact address}

Larry Ching Fan Li

Tan Orthodontics, 174 East Boundary Road,

Bentleigh, Victoria 3165, Austrália

E-mail: larrycfli@yahoo.com 